\title{
Oridonin Attenuates Lipopolysaccharide-Induced ROS Accumulation and Inflammation in HK-2 Cells
}

\author{
Jen-Hsuan Huang $\mathbb{D}^{1},{ }^{1}$ Chou-Chin Lan $\mathbb{D}^{2,3}$ Ya-Ting Hsu ${ }^{2},{ }^{4}$ Cheng-Lin Tsai ${ }^{4}{ }^{4}$ \\ I-Shiang Tzeng $\mathbb{D},{ }^{5}$ Po Wang $\mathbb{D},{ }^{5}$ Chan-Yen Kuo $\mathbb{D},,^{5}$ and Po-Chun Hsieh $\mathbb{D}^{4}$ \\ ${ }^{1}$ Department of Anesthesiology, Show Chwan Memorial Hospital, Changhua, Taiwan \\ ${ }^{2}$ Division of Pulmonary Medicine, Taipei Tzu Chi Hospital, Buddhist Tzu Chi Medical Foundation, New Taipei, Taiwan \\ ${ }^{3}$ School of Medicine, Tzu Chi University, Hualien, Taiwan \\ ${ }^{4}$ Department of Chinese Medicine, Taipei Tzu Chi Hospital, Buddhist Tzu Chi Medical Foundation, New Taipei City, Taiwan \\ ${ }^{5}$ Department of Research, Taipei Tzu Chi Hospital, Buddhist Tzu Chi Medical Foundation, New Taipei City, Taiwan
}

Correspondence should be addressed to Chan-Yen Kuo; cykuo863135@gmail.com and Po-Chun Hsieh; pchsieh.tcm@gmail.com

Received 23 July 2019; Revised 6 December 2019; Accepted 8 January 2020; Published 27 February 2020

Academic Editor: Manel Santafe

Copyright ( 92020 Jen-Hsuan Huang et al. This is an open access article distributed under the Creative Commons Attribution License, which permits unrestricted use, distribution, and reproduction in any medium, provided the original work is properly cited.

\begin{abstract}
Renal tubulointerstitial inflammation plays an important role in chronic kidney disease (CKD). Inflammation reduction is a good strategy to combat CKD. Oridonin, an ent-kaurane diterpenoid isolated from Rabdosia rubescens (Donglingcao), is considered as an effective natural candidate for the treatment of anti-inflammatory, antiviral, and antibacterial activities, including liver fibrosis and many tumors; however, no study has demonstrated its effect on lipopolysaccharide- (LPS-) induced renal inflammation. To investigate the anti-inflammatory effects of oridonin on human renal proximal tubular epithelial cells (HK-2 cells), the expression levels of c-Jun $\mathrm{N}$-terminal kinase (JNK) and reactive oxygen species (ROS) were evaluated by Western blot analysis and $2^{\prime}, 7^{\prime}$-dichlorofluorescein diacetate (DCF-DA) staining, respectively. The level of intracellular ROS increased in a dose-dependent manner following LPS treatment, whereas oridonin inhibited this effect, suggestive of its ability to prevent ROS accumulation. As the mitogen-activated protein kinase (MAPK) family of enzymes plays an important role in physiological responses, we examined the activation of JNK by Western blotting and found that oridonin attenuated LPS-induced JNK phosphorylation. Oridonin also attenuated RAW 264.7 cell chemotaxis towards LPS-treated HK-2 cells. Taken together, oridonin protected against LPS-induced inflammation including ROS accumulation, JNK activation, NF- $\kappa$ B nuclear translocation in HK-2 cells, and functionally blocked macrophage chemotaxis towards LPS-treated HK-2 cells. Oridonin may exhibit therapeutic potential by the anti-inflammation effect in LPS-treated HK-2 cells.
\end{abstract}

\section{Introduction}

Chronic kidney disease (CKD) is characterized by a progressive and irreversible exacerbation of the renal excretory function that necessitates renal replacement therapy in the form of dialysis or renal transplants and may also lead to death [1]. Therefore, CKD is a global health problem with serious implications. Renal tubulointerstitial inflammation has an important role in fibrosis, which is the key pathogenetic alteration associated with CKD [2].

Wang et al. suggested that an increase in oxidative stress plays a critical role in mediating metabolic syndrome-induced tubulointerstitial injury [3]. Albuminuria may serve as an endogenous danger-associated molecular pattern (DAMP) that stimulates tubulointerstitial inflammation via mitochondrial reactive oxygen species- (mtROS-) mediated activation of the cytoplasmic Nlrp3 inflammasome in HK-2 cells [4]. Evidence indicates that SIRT3, an antiaging molecule regulated by calorie restriction and mitochondrionlocalized NAD (+) -dependent deacetylase, positively regulates both mitochondrial oxidative capacity and antioxidant gene expression, thereby reducing ROS accumulation in mouse proximal tubular cells [5]. It is well known that activated macrophages respond to inflammation induced by bacterial lipopolysaccharide (LPS) by producing cytokines (interferon-gamma, granulocyte-monocyte colony- 
stimulating factor, and tumor necrosis factor-alpha), extracellular matrix proteins, and other chemical mediators [6]. LPS plays a crucial role in macrophage infiltration and progressive chronic kidney inflammatory injury and fibrosis via activation of the mammalian target of rapamycin(mTOR-) dependent pathway [7]. However, the pharmacological effects of oridonin and the underlying mechanism of action in renal tubulointerstitial inflammation are still unknown.

Oridonin, an ent-kaurane diterpenoid isolated from Rabdosia rubescens (Donglingcao), has various pharmacological and biological properties such as anti-inflammatory, antiviral, and antibacterial activities [8]. Many studies have suggested that oridonin has potent anticancer activity against several cancers, including lung cancer, colorectal cancer, and hepatocellular carcinoma, in vitro and in vivo [9-12] and may exert beneficial effect against Alzheimer's disease [13], acute lung injury [14], postinflammatory irritable bowel syndrome [15], and Crohn's disease [16]. These diseases are closely related to inflammation. It is known that mtROS are signaling molecules involved in the physiological recruitment of patrolling cells and pathological recruitment of inflammatory cells [17]. ROS accumulation ultimately leads to DNA damage and cell death via glutathione depletion and caspase-3 activation $[18,19]$. Therefore, it is important to identify the candidate compounds that respond to the inflammation caused by ROS overproduction during the development of renal tubulointerstitial disease.

Macrophages are important tumor-infiltrating cells and play critical roles in tumorigenesis and metastasis [20]. Liu et al. reported that LPS induces chemokines secretions, including interleukin-8 (IL-8), monocyte chemoattractant protein-1 (MCP-1), and macrophage inflammatory protein$1 \alpha(\mathrm{MIP}-1 \alpha)[21]$. Therefore, a survey of candidate compounds with potential antichemotaxis effects on RAW 264.7 cell, as reported by Kuo et al. in a previous study, is needed [22].

This is the first study aiming for investigating the antiinflammatory effects of oridonin on LPS stimulated HK-2 cells and the underlying mechanisms of action, including ROS accumulation, c-Jun N-terminal kinase (JNK) phosphorylation, nuclear factor kappa $B(N F-\kappa B)$ nuclear translocation, and RAW 264.7 cell chemotaxis.

\section{Materials and Methods}

2.1. Reagent. Oridonin (CAS 28957-04-2) was purchased from Merck (MO, USA). The HPLC description was $\geq 93 \%$.

2.2. Antibodies. The following antibodies were used for immunofluorescence staining and Western blotting: rabbit polyclonal antibodies to p-JNK (ABclonal, MA, USA), JNK (ABclonal, MA, USA), $\beta$-actin (ABclonal, MA, USA), p65 (Cell signaling, MA, USA), fibrillarin (Cell signaling, MA, USA), Cox-2 (ABclonal, MA, USA), iNOS (Abcam, UK), CTGF (ABclonal, MA, USA), and $a$-SMA (ABclonal, MA, USA).
2.3. Cell Culture. Human renal proximal tubular epithelial cells (HK-2) were purchased from Bioresource Collection and Research Center (BCRC, Taiwan) and cultured in T-75 flasks (Corning, NY, USA) in Dulbecco's modified Eagle's medium (DMEM; Ham's F12 (Gibco, NY, USA) supplemented with $10 \%$ fetal bovine serum (FBS), $25 \mathrm{mM}$ D-glucose, $2 \mathrm{mM}$ L-glutamine, $1 \mathrm{mM}$ sodium pyruvate, and penicillin-streptomycin (50 U/mL; Sigma, MO, USA) at $37^{\circ} \mathrm{C}$ in $5 \% \mathrm{CO}_{2} / 95 \%$ air. The culture medium was replaced with fresh medium on alternate days. The cells were trypsinized once they reached $70 \%$ confluence and used for subsequent experiments.

The murine monocyte/macrophage cell line RAW 264.7 (BCRC \#60001) was purchased from BCRC (Taiwan). RAW 264.7 cell line was cultured in DMEM supplemented with $10 \%$ FBS, $4 \mathrm{mM}$ L-glutamine, $4,500 \mathrm{mg} / \mathrm{L}$ glucose, $1 \mathrm{mM}$ sodium pyruvate, $1,500 \mathrm{mg} / \mathrm{L}$ sodium bicarbonate, and $50 \mathrm{U} /$ $\mathrm{mL}$ penicillin-streptomycin (Sigma-Aldrich, St. Louis, MO, USA) at $37^{\circ} \mathrm{C}$ in $5 \% \mathrm{CO}_{2} / 95 \%$ air for no more than five passages. The culture medium was replaced on alternate days. The cells were collected via trypsinization after reaching 50\%$60 \%$ confluence and used for subsequent experiments.

2.4. Western Blot Analysis. The Western blot analysis was according to our previous studies with some modifications [23]. Total proteins were extracted from HK-2 cells using ice-cold radioimmunoprecipitation assay (RIPA) buffer (20 mM Tris-HCl [pH 7.4], $150 \mathrm{mM}$ sodium chloride (NaCl), $1 \mathrm{mM}$ EGTA, $1 \mathrm{mM}$ sodium fluoride $(\mathrm{NaF})), 2 \mathrm{mM}$ sodium orthovanadate $\left(\mathrm{Na}_{3} \mathrm{VO}_{4}\right), 1 \mathrm{mM}$ phenylmethylsulphonyl fluoride, $1 \%$ dilution of Sigma protease cocktail, and $1 \%$ Triton X-100). Extracted proteins were separated by $10 \%$ or $15 \%$ sodium dodecyl sulfate-polyacrylamide gel electrophoresis (SDS-PAGE), and the separated protein bands were transferred onto nitrocellulose membranes. Immunoblotting was performed using specific primary antibodies and horseradish peroxidase-conjugated secondary antibodies (Cell Signaling Technology, MA, USA). Peroxidase activity was assessed using an enhanced chemiluminescence detection kit (PerkinElmer Life Science, MA, USA).

2.5. Measurement of Intracellular ROS Generation. The analysis of the measurement of intracellular ROS generation was according to our previous studies with some modifications [23]. Cells were washed with phosphate-buffered saline (PBS) and incubated with $10 \mu \mathrm{M}$ of $2^{\prime}, 7^{\prime}$-dichlorofluorescein diacetate (DCF-DA; Sigma, MO, USA) at $37^{\circ} \mathrm{C}$ for $30 \mathrm{~min}$ in the dark. In the presence of ROS, DCF-DA is oxidized to produce fluorescence. After incubation, the cells were trypsinized and washed thrice with ice-cold PBS. The ROS level was quantified by flow cytometry (BD Biosciences, CA, USA) using $488 \mathrm{~nm}$ excitation/585 nm emission filters.

2.6. Cell Viability Assay. We used WST-1 assay (Abcam, $\mathrm{UK})$ to detect the cell viability, according to the manufacturer's instructions. In brief, HK-2 cells were seeded at a 
density of $5 \times 10^{4}$ cells $/ \mathrm{ml}$ in 24 -well plates and cultured in phenol red-free DMEM containing $0.5 \%$ heat-inactivated FBS for $24 \mathrm{~h}$. After incubation, cells were incubated with indicated concentrations of LPS for $24 \mathrm{~h}$. The WST- 1 reagent was then added to the medium and incubated at $37^{\circ} \mathrm{C}$ for $2 \mathrm{~h}$. The absorbance was measured at $450 \mathrm{~nm}$ in a microplate reader (BIO-RAD, CA, USA).

2.7. Chemotaxis Assay. The chemotaxis analysis was according to our previous studies with some modifications [23]. We used 24-well Transwell plates $(8 \mu \mathrm{m}$ pore size; Corning, NY, USA) to measure chemotaxis of RAW 264.7 cells. HK-2 cells were preconditioned with or without $30 \mu \mathrm{M}$ oridonin for $12 \mathrm{~h}$ before incubation with 1,3 , or $10 \mu \mathrm{g} / \mathrm{mL}$ of LPS for $24 \mathrm{~h}$. These cells were seeded into the lower chamber for $24 \mathrm{~h}$ in serum-free media supplemented with 5\% bovine serum albumin (Sigma-Aldrich, MO, USA). RAW 264.7 cells $\left(1 \times 10^{5}\right)$ were seeded in the upper chamber and placed over the lower chamber containing treated or untreated HK-2 cells. The upper chamber containing RAW 264.7 cells was used for the chemotaxis assay. The migratory cells on the lower side of the membrane were stained with $0.1 \%$ crystal violet for $5 \mathrm{~min}$ and washed with water; the membrane was scanned using EPSON V750 PRO scanner. Crystal violet was destained with methanol for $15 \mathrm{~min}$, and the absorbance values were measured at OD570. All measured values were detected by Synergy HT (BioTek, VT, USA).

2.8. Nuclear Fraction Extraction. The nuclear fraction extraction was according to our previous studies with some modifications [23]. The nuclear fraction was extracted from HK-2 cells. The cells were collected and resuspended in a hypotonic buffer (10 mM HEPES, pH 7.9, $10 \mathrm{mM}$ ) potassium chloride $(\mathrm{KCl}), 1.5 \mathrm{mM}$ magnesium chloride $\left(\mathrm{MgCl}_{2}\right)$, $0.2 \mathrm{mM}$ phenylmethylsulphonyl fluoride (PMSF), $20 \mu \mathrm{g} / \mathrm{mL}$ aprotinin, $0.5 \mathrm{mM}$ dithiothreitol (DTT), and $0.5 \% \mathrm{NP}-40$ on ice for $15 \mathrm{~min}$. After centrifugation at $6,000 \times g$ for $15 \mathrm{~min}$ at $4^{\circ} \mathrm{C}$, the pellet was collected and washed with a basal buffer (hypotonic buffer without $0.5 \% \mathrm{NP}-40$ ). The cells were centrifuged at $6,000 \times g$ for $15 \mathrm{~min}$ at $4^{\circ} \mathrm{C}$, and the pellet was collected and resuspended in a hypertonic buffer $(20 \mathrm{mM}$ HEPES, pH 7.9, $400 \mathrm{mM} \mathrm{KCl}, 1.5 \mathrm{mM} \mathrm{MgCl}_{2}, 0.2 \mathrm{mM}$ PMSF, $20 \mu \mathrm{g} / \mathrm{mL}$ aprotinin, $0.5 \mathrm{mM}$ DTT, $0.2 \mathrm{mM}$ ethylenediaminetetraacetic acid (EDTA), and 10\% glycerol) at $25^{\circ} \mathrm{C}$ for $30 \mathrm{~min}$. After centrifugation at $10,000 \times g$ for $30 \mathrm{~min}$ at $4^{\circ} \mathrm{C}$, the nuclear fraction in the supernatant was collected.

2.9. Statistical Analysis. Statistical analysis was performed with IBM SPSS Statistics 25 (IBM, NY, USA). Data are expressed as means \pm standard deviation. Groups were compared with one-way or two-way analysis of variance (ANOVA) followed by Bonferroni post hoc analysis. A value of $p<0.05$ was considered to indicate statistical significance.

\section{Results}

3.1. LPS-Induced ROS Accumulation, JNK Phosphorylation, and NF- $\kappa B$ Nuclear Translocation in HK-2 Cells. We observed an increase in ROS production after LPS stimulation at the indicated dose (Figure 1(a)). To detect the effect of LPS on cell viability, the WST-1 was used in indicated concentrations $(0,1,3$, and $10 \mu \mathrm{g} / \mathrm{mL})$ of LPS-treated HK-2 cells. Results demonstrated that the cell viability was not changed even in the highest concentration $(10 \mu \mathrm{g} / \mathrm{mL})$ of LPS (Figure 1(b)). The JNK pathway is activated by stress and inflammatory signals [24]. To investigate the inflammatory effect of LPS on HK-2 cells, we evaluated JNK phosphorylated and NF- $\kappa \mathrm{B}$ nuclear translocation by Western blot analysis. The result obtained indicated that LPS-induced JNK phosphorylation (JNK pathway activation) without alerting in the total JNK protein levels (Figures 2(a) and $2(\mathrm{~b})$ ) and induced the nuclear translocation of NF- $\kappa \mathrm{B}$ p 65 (Figure 3, lane 6).

3.2. LPS-Induced RAW 264.7 Cell Chemotaxis. To confirm the inflammatory effects caused by LPS, we devised an in vitro microenvironment to examine macrophage chemotaxis following LPS stimulation of HK-2 cells. We observed that groups treated with 1,3 , or $10 \mu \mathrm{g} / \mathrm{mL}$ LPS (Figures 4 (a) and 4(b), column 2,3) showed a greater than twofold increase in macrophage chemotaxis as compared with the untreated group (Figures 4(a) and 4(b), column 1).

3.3. Oridonin Attenuated LPS-Induced HK-2 Inflammation. We evaluated the effects of oridonin on LPS-induced ROS production, JNK phosphorylation, NF- $\kappa \mathrm{B}$ nuclear translocation in HK-2 cells, and RAW 264.7 chemotaxis. The results obtained indicated that oridonin treatment attenuated LPSinduced ROS production (Figure 5, column 3, 4), JNK phosphorylation (Figure 6, lanes 2, 4), and nuclear translocation of NF- $\kappa \mathrm{B}$ p65 (Figure 3, lanes 6, 8). Oridonin also inhibited LPS-induced RAW 264.7 cells chemotaxis (Figure 4, columns 3, 4).

To further confirm the anti-inflammatory effect of oridonin on LPS-treated HK-2 cells, we evaluated the expressions of iNOS and Cox- 2 by Western blotting. Results demonstrated that oridonin treatment attenuated LPS-induced increasing in iNOS and Cox-2. These findings supported that oridonin has an anti-inflammatory effect on LPS-induced HK-2 cell inflammation in vitro (Figure 7).

3.4. Oridonin Attenuated LPS-Induced HK-2 Fibrotic Effect. To further confirm the antifibrotic-related effect of oridonin on LPS-treated HK-2 cells, we evaluated the expressions of CTGF and a-SMA by Western blotting. Results demonstrated that oridonin treatment attenuated LPS-induced increasing in CTGF and $a$-SMA. These findings supported that oridonin has an antifibrotic effect on LPS-induced renal fibrosis in vitro (Figure 7). 


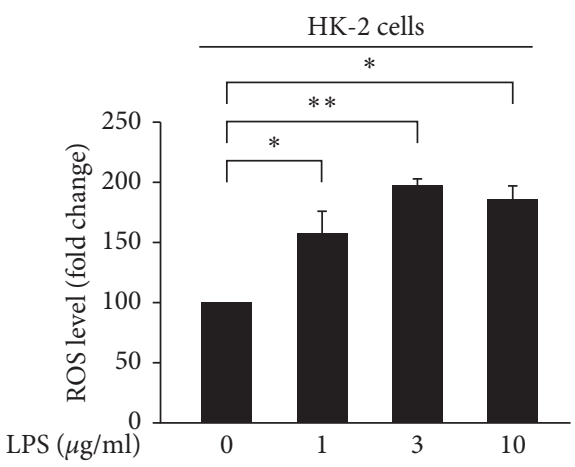

(a)

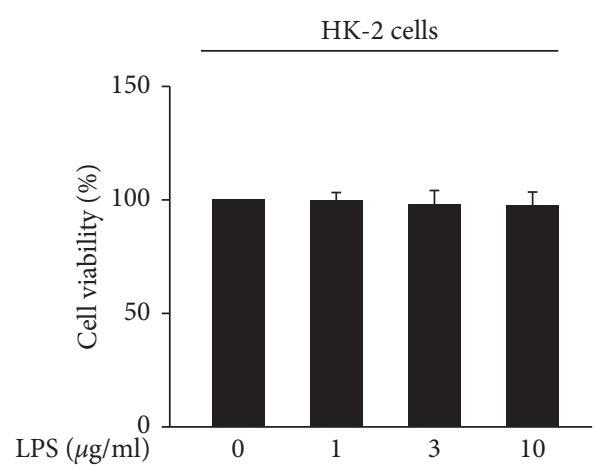

(b)

FIGURE 1: LPS-induced ROS accumulation. (a) HK-2 cells were treated with either the control or indicated concentrations of LPS for $24 \mathrm{~h}$ The levels of intracellular ROS were determined using DCF-DA, while the fluorescence signal was detected using a FACSCalibur instrument. (b) After the incubation period, cell viability was determined using WST-1 assay. All data are presented as the mean \pm SD. $n=3 .{ }^{*} p<0.05$, ${ }^{* *} p<0.01$.

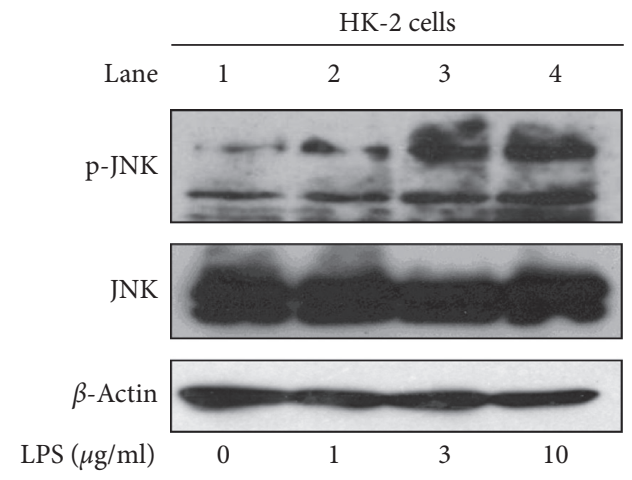

(a)

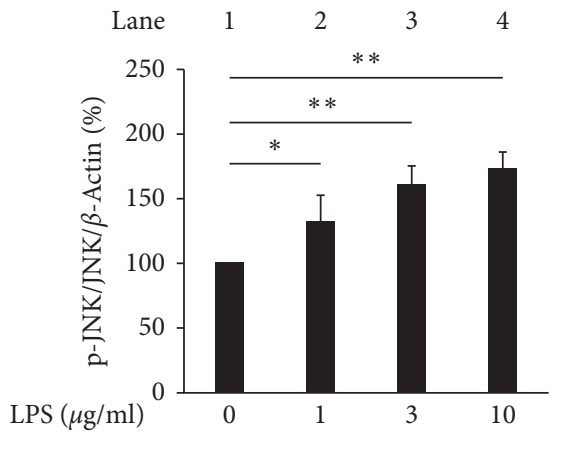

(b)

FIGURE 2: LPS-induced phosphorylation of JNK. (a) LPS-induced phosphorylation of JNK in a dose-dependent manner. HK-2 cells were treated with LPS at the indicated concentrations for $24 \mathrm{~h}$. Phosphorylation of JNK was analyzed using immunoblotting analysis with antibodies against phosphorylated and total protein. (b) Densitometric analysis of all samples normalized against the level of total protein. All data are presented as mean \pm SD. $n=3 .{ }^{*} p<0.05,{ }^{* *} p<0.01$.

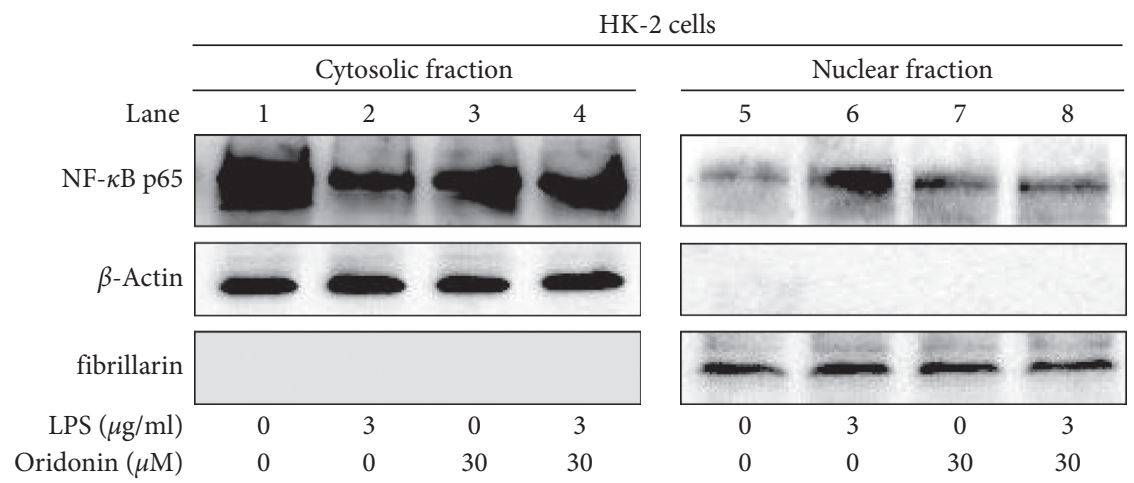

FIGURE 3: Oridonin attenuated LPS-induced NF- $\kappa$ B nuclear translocation. HK-2 cells cultured and treated as above were collected, and cytosolic and nuclear fractions were isolated as described in Materials and Methods. Western blot analysis was performed to detect the subcellular localization of NF- $\kappa \mathrm{B}$ using an antibody against the NF- $\kappa \mathrm{B}$ subunit p65. $\beta$-actin was used as a cytosolic marker and fibrillarin served as a nuclear marker. 


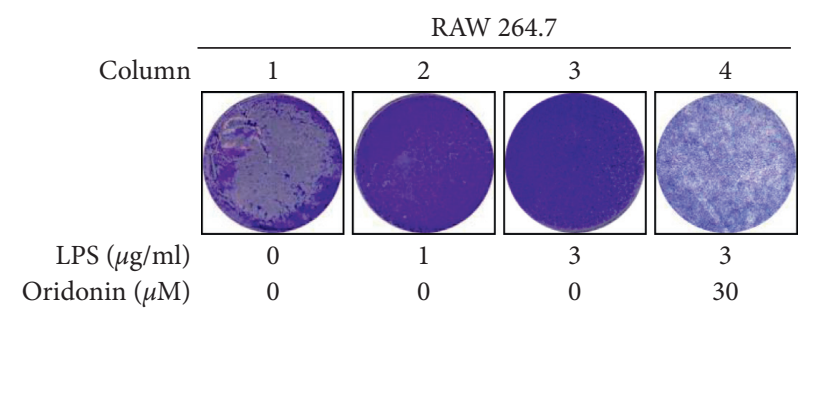

(a)

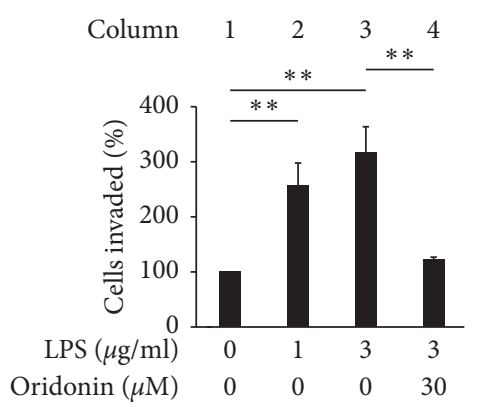

(b)

FIGURE 4: Effects of LPS on the recruitment of monocyte/macrophage RAW 264.7 cells with or without oridonin treatment. HK-2 cells with or without LPS treatment were assayed for their ability to recruit monocyte/macrophage cells (RAW 264.7) in the presence or absence of oridonin. The details are described in Materials and Methods. (a) Macroscopic observation of Transwell chambers (upper). (b) Relative levels of migrated cells presented as the extent of crystal violet staining measured at OD570. All data are presented as mean \pm SD. $n=3$. ${ }^{* *} p<0.01$.

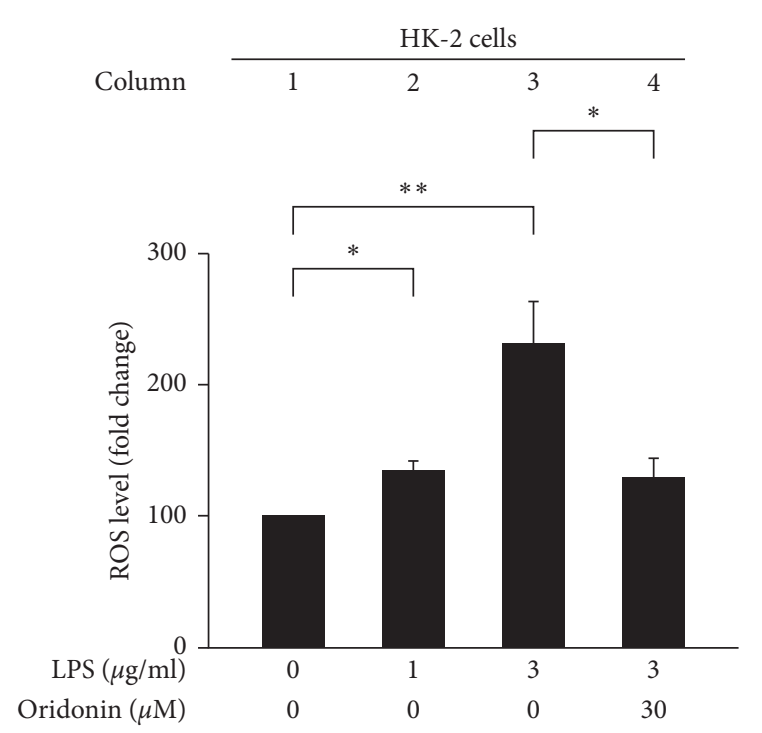

FIGURE 5: Oridonin attenuated ROS accumulation in LPS-treated HK-2 cells. HK-2 cells were pretreated with oridonin for $12 \mathrm{~h}$ before the addition of LPS. The levels of intracellular ROS were determined using DCF-DA, and the fluorescence was detected using FACSCalibur analysis. All data are presented as mean \pm SD. $n=3$. ${ }^{*} p<0.05,{ }^{* *} p<0.01$.

\section{Discussion}

In the present study, oridonin preconditioning exhibited protective effects on LPS-induced iNOS expression, ROS accumulation, JNK phosphorylation, and NF- $\kappa \mathrm{B}$ nuclear translocation, COX-2, $a$-SMA, and CTGF expression in HK2 cells. Oridonin also inhibited RAW 264.7 chemotaxis. To the best of our knowledge, this is the first study to explore the role of oridonin in LPS-induced ROS accumulation, inflammation, and fibrotic effect in HK-2 cells and RAW 264.7 cell chemotaxis.

Several studies have proposed that the JNK, p38, and extracellular signal regulated kinase (ERK) signaling pathways are involved in LPS-induced inflammatory cytokine production [24, 25]. Activation of JNK signaling is a common phenomenon in most forms of human kidney injury, evident in both intrinsic glomerular and tubular cells as well as in infiltrating leukocytes. Similar patterns of JNK activation are evident in animal models of acute and chronic renal injury [26]. NF- $\kappa \mathrm{B}$ nuclear translocation is an indicator of chronic inflammation and ROS accumulation during the progression and development of CKD [27, 28]. Therefore, the inhibition of inflammation and oxidative stress may serve as a good strategy for the treatment of CKD. Su et al. reported the renal protective effect of Fangjifuling against LPS-induced inflammatory and apoptotic responses in vitro and in vivo [29]. Similar protective effects were reported for berberine against chronic renal failure mediated via tumor necrosis factor receptor associated factor 5- (TRAF5-) induced activation of the NF- $\kappa \mathrm{B}$ signaling pathway in mouse podocytes [30]. Consistent with the results of the previous studies [28, 31], we proposed that traditional Chinese medicine regularly used in clinical patients may provide protective effects against kidney injury.

Oridonin exerts protective effects against diabetes-induced renal injury via the Toll-like receptor 4 (TLR4)/p38mitogen-activated protein kinase (MAPK) and NF- $\kappa \mathrm{B}$ signaling pathways [32]. Furthermore, oridonin enhanced 5-fluorouracil-triggered 786-O cell necroptosis via the ROS-dependent but JNK-, p38-, and ERK-independent, cascades. This effect suggests its potential application in combination with chemotherapeutic agents for cancer treatment [33, 34].

Macrophage activation depends on the surrounding microenvironment and heterogeneous cell populations that are present in all tissues. Tumor-associated macrophages (TAMs) may be divided into classically activated inflammatory macrophages (M1) and alternatively activated antiinflammatory macrophages (M2) [35]. LPS has been reported to activate M1 macrophages via NF- $\kappa$ B nuclear translocation [36]. Consistent with our findings (Figure 4), it has been reported that the 4-O-methylhonokiol analog GS12021 inhibits TNF- $\alpha$ inflammation and macrophage chemotaxis [37]. We propose that oridonin may exert protective effects on macrophage infiltration. Hence, the anti-inflammation effects may contribute to alleviating RAW 264.7 chemotaxis. Our results suggest that oridonin treatment may serve as a feasible treatment strategy for 


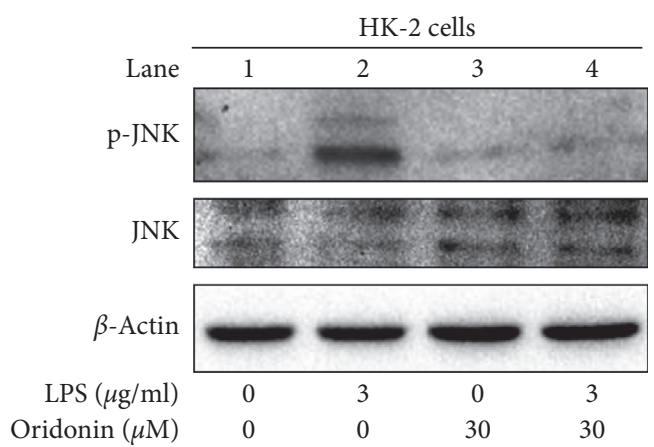

(a)

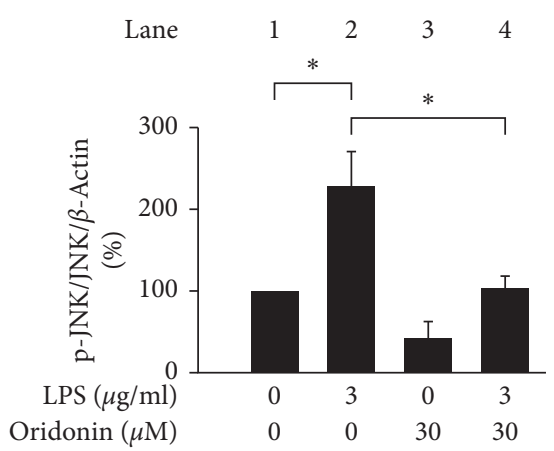

(b)

FIGURE 6: Oridonin attenuated LPS-induced phosphorylation of JNK. (a) HK-2 cells were pretreated with oridonin (30 $\mu$ M) for $12 \mathrm{~h}$ before the addition of $3 \mu \mathrm{g} / \mathrm{mL}$ LPS. Phosphorylation of JNK was analyzed by immunoblotting using antibodies against phosphorylated and total protein. (b) Densitometric analysis of all samples normalized against the level of total protein. All data are presented as mean \pm SD. $n=3$. $* p<0.05$.

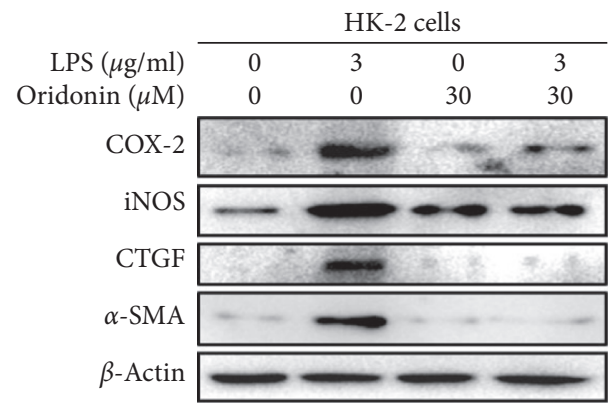

(a)

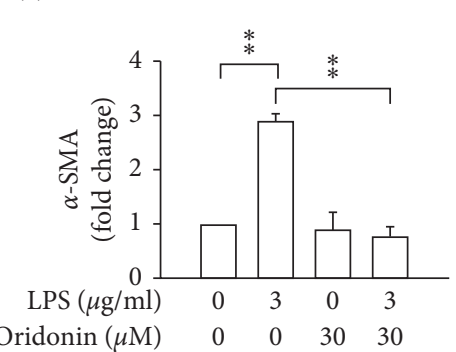

(d)

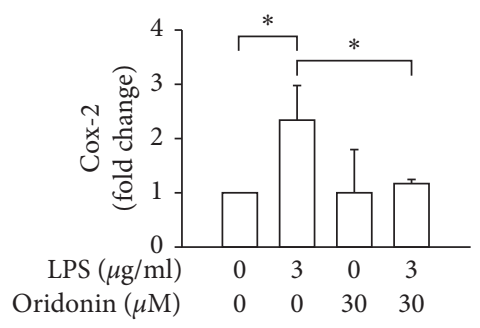

(b)

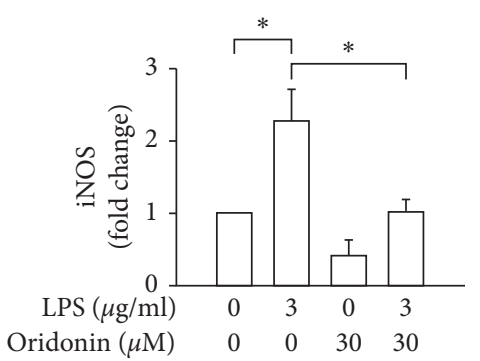

(c)

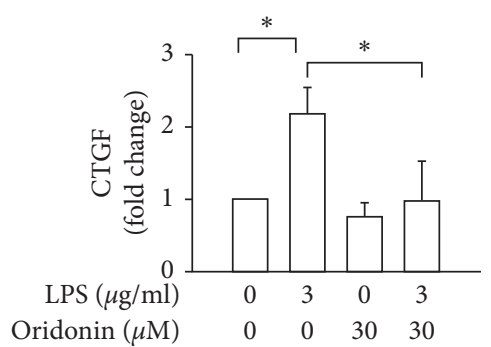

(e)

FIGURE 7: Oridonin attenuated LPS-induced iNOS, COX-2, a-SMA, and CTGF expression. HK-2 cells were pretreated with oridonin $(30 \mu \mathrm{M})$ for $12 \mathrm{~h}$ before the addition of $3 \mu \mathrm{g} / \mathrm{mL}$ LPS. iNOS, COX-2, a-SMA, and CTGF were analyzed by immunoblotting. (b-e) Densitometric analysis of all samples normalized against the level of total protein. All data are presented as mean \pm SD. $n=3$. ${ }^{*} p<0.05$, ${ }^{* * *} p<0.01$.

kidney injury, particularly concerning inflammatory responses.

The inflammatory response is closely associated with JNK phosphorylation activated by cytokines, nutrients, growth factors, and physical-chemical-mechanical stress [38]. Therefore, inhibition of JNK by targeting myeloid cells with a drug may provide therapeutic benefits for the treatment of inflammation-related liver diseases [39]. In the present study, the results indicated that oridonin attenuated LPS-induced HK-2 cells JNK phosphorylation (Figure 6, lane 2,4 ), NF- $\kappa \mathrm{B}$ activation (NF- $\kappa \mathrm{B}$ nuclear translocation, Figure 3 lane 6, 8), and ROS accumulation (Figure 5, column $3,4)$ in HK-2 cells. We propose that inflammation is a systemic response dependent on various signaling pathways, consistent with a previous study by Chen et al. [40].

In summary, our results demonstrated that oridonin preconditioning exhibited protective effects on LPS-induced iNOS expression, ROS accumulation, JNK phosphorylation, and NF- $\kappa$ B nuclear translocation, COX-2, $a$-SMA, and CTGF expression in HK-2 cells (Figure 8). Oridonin also inhibited RAW 264.7 chemotaxis (Figure 9). These novel findings should deepen our understanding of the mechanistic action of oridonin. Since oridonin has been proven to attenuate LPS-induced inflammation in HK-2 cells and inhibit RAW 264.7 cell chemotaxis, further in vivo study based on Vhlh conditional knockout mice to mimic chronic 


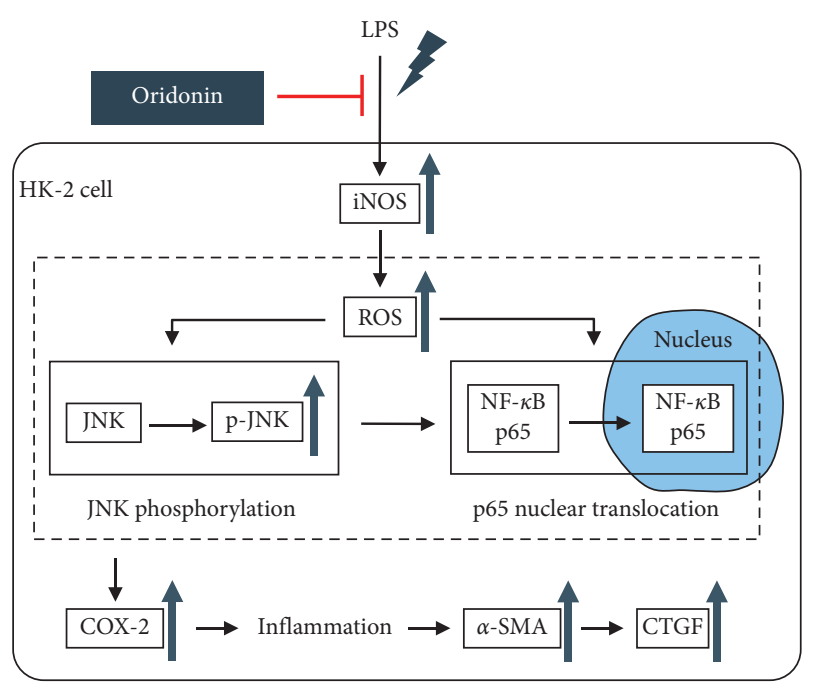

FIGURE 8: Anti-inflammation and antifibrotic mechanisms of oridonin. Oridonin preconditioning exhibited protective effects on LPS-induced iNOS expression, ROS accumulation, JNK phosphorylation, and NF- $\kappa \mathrm{B}$ nuclear translocation, COX-2, $a$-SMA, and CTGF expression in HK-2 cells.

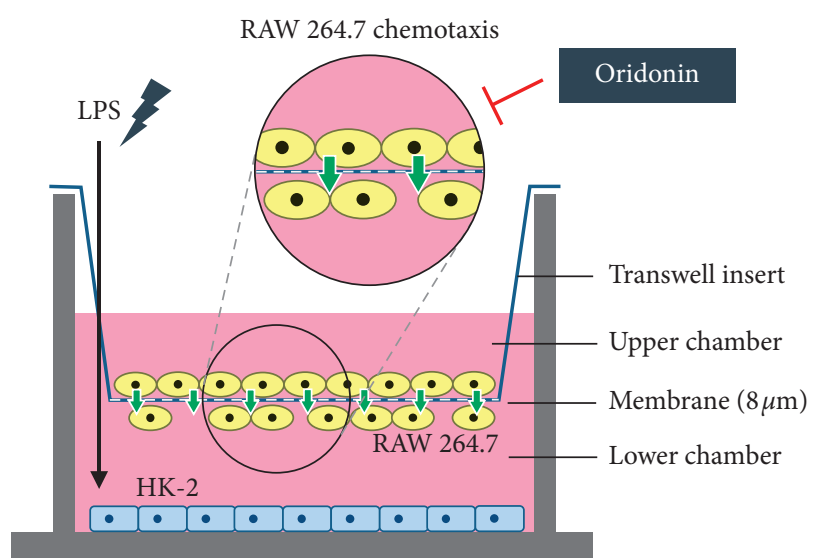

FIGURE 9: Antichemotaxis mechanisms of oridonin. Oridonin inhibited RAW 264.7 cell chemotaxis.

kidney inflammation in our previous study [23] is required to determine the potential effects of oridonin on human renal proximal tubular epithelial cells in individuals with CKD.

\section{Data Availability}

The original data used to support the findings of this study are included in the article.

\section{Conflicts of Interest}

The authors declare no conflicts of interest.

\section{Acknowledgments}

This study was supported by grants RD 107052 from Show Chwan Memorial Hospital, Changhua, Taiwan, and TCRD-
TPE-109-06 (1/2) from Taipei Tzu Chi Hospital, Buddhist Tzu Chi Medical Foundation, New Taipei City, Taiwan.

\section{References}

[1] J. M. López-Novoa, C. Martínez-Salgado, A. B. RodríguezPeña, and F. J. L. Hernández, "Common pathophysiological mechanisms of chronic kidney disease: therapeutic perspectives," Pharmacology \& Therapeutics, vol. 128, no. 1, pp. 61-81, 2010.

[2] A. Nogueira, M. J. Pires, and P. A. Oliveira, "Pathophysiological mechanisms of renal fibrosis: a review of animal models and therapeutic strategies," In Vivo, vol. 31, no. 1, pp. 1-22, 2017.

[3] C. Wang, E. R. Blough, R. Arvapalli et al., "Metabolic syndrome-induced tubulointerstitial injury: role of oxidative stress and preventive effects of acetaminophen," Free Radical Biology and Medicine, vol. 65, pp. 1417-1426, 2013.

[4] D. Liu, M. Xu, L.-H. Ding et al., "Activation of the Nlrp3 inflammasome by mitochondrial reactive oxygen species: a novel mechanism of albumin-induced tubulointerstitial inflammation," The International Journal of Biochemistry \& Cell Biology, vol. 57, pp. 7-19, 2014.

[5] T. Koyama, S. Kume, D. Koya et al., "SIRT3 attenuates palmitate-induced ROS production and inflammation in proximal tubular cells," Free Radical Biology and Medicine, vol. 51, no. 6, pp. 1258-1267, 2011.

[6] N. Fujiwara and K. Kobayashi, "Macrophages in inflammation," Current Drug Target -Inflammation \& Allergy, vol. 4, no. 3, pp. 281-286, 2005.

[7] H. Chen, J. Zhu, Y. Liu et al., "Lipopolysaccharide induces chronic kidney injury and fibrosis through activation of mTOR signaling in macrophages," American Journal of $\mathrm{Ne}$ phrology, vol. 42, no. 4, pp. 305-317, 2015.

[8] Y. Xu, Y. Xue, Y. Wang, D. Feng, S. Lin, and L. Xu, "Multiplemodulation effects of Oridonin on the production of proinflammatory cytokines and neurotrophic factors in LPSactivated microglia," International Immunopharmacology, vol. 9, no. 3, pp. 360-365, 2009.

[9] W. Tian and S.-y. Chen, "Recent advances in the molecular basis of anti-neoplastic mechanisms of oridonin," Chinese Journal of Integrative Medicine, vol. 19, no. 4, pp. 315-320, 2013.

[10] H. Jin, X. Tan, X. Liu, and Y. Ding, "Downregulation of AP-1 gene expression is an initial event in the oridonin-mediated inhibition of colorectal cancer: studies in vitro and in vivo," Journal of Gastroenterology and Hepatology, vol. 26, no. 4, pp. 706-715, 2011.

[11] H. Wang, Y. Ye, S. Y. Pan et al., "Proteomic identification of proteins involved in the anticancer activities of oridonin in HepG2 cells," Phytomedicine: International Journal of Phytotherapy and Phytopharmacology, vol. 18, no. 2-3, pp. 163169, 2011.

[12] H.-Y. Kwan, Z. Yang, W.-F. Fong, Y.-M. Hu, Z.-L. Yu, and W.-L. W. Hsiao, "The anticancer effect of oridonin is mediated by fatty acid synthase suppression in human colorectal cancer cells," Journal of Gastroenterology, vol. 48, no. 2, pp. 182-192, 2013.

[13] E.-J. Seo, N. Fischer, and T. Efferth, "Phytochemicals as inhibitors of NF- $\kappa \mathrm{B}$ for treatment of Alzheimer's disease," Pharmacological Research, vol. 129, pp. 262-273, 2018.

[14] G. Zhao, T. Zhang, X. Ma et al., "Oridonin attenuates the release of pro-inflammatory cytokines in lipopolysaccharide- 
induced RAW264.7 cells and acute lung injury," Oncotarget, vol. 8, no. 40, pp. 68153-68164, 2017.

[15] K.-h. Zang, Y.-y. Shao, X. Zuo, Z. Rao, and H.-y. Qin, "Oridonin alleviates visceral hyperalgesia in a rat model of postinflammatory irritable bowel syndrome: role of colonic enterochromaffin cell and serotonin availability," Journal of Medicinal Food, vol. 19, no. 6, pp. 586-592, 2016.

[16] S. Wang, Y. Zhang, P. Saas et al., "Oridonin's therapeutic effect: suppressing Th1/Th17 simultaneously in a mouse model of Crohn's disease," Journal of Gastroenterology and Hepatology, vol. 30, no. 3, pp. 504-512, 2015.

[17] X. Li, P. Fang, W. Y. Yang et al., "Mitochondrial ROS, uncoupled from ATP synthesis, determine endothelial activation for both physiological recruitment of patrolling cells and pathological recruitment of inflammatory cells," Canadian Journal of Physiology and Pharmacology, vol. 95, no. 3, pp. 247-252, 2017.

[18] H. Blaser, C. Dostert, T. W. Mak, and D. Brenner, "TNF and ROS crosstalk in inflammation," Trends in Cell Biology, vol. 26, no. 4, pp. 249-261, 2016.

[19] S. Dunning, A. Ur Rehman, M. H. Tiebosch et al., "Glutathione and antioxidant enzymes serve complementary roles in protecting activated hepatic stellate cells against hydrogen peroxide-induced cell death," Biochimica et Biophysica Acta (BBA)-Molecular Basis of Disease, vol. 1832, no. 12, pp. 2027-2034, 2013.

[20] Y.-C. Wang, F. He, F. Feng et al., "Notch signaling determines the M1 versus M2 polarization of macrophages in antitumor immune responses," Cancer Research, vol. 70, no. 12, pp. 4840-4849, 2010.

[21] Y. Liu, W.-W. Su, S. Wang, and P.-B. Li, "Naringin inhibits chemokine production in an LPS-induced RAW 264.7 macrophage cell line," Molecular Medicine Reports, vol. 6, no. 6, pp. 1343-1350, 2012.

[22] L.-M. Kuo, P.-Y. Tseng, Y.-C. Lin et al., "New hirsutinolidetype sesquiterpenoids from vernonia cinerea inhibit nitric oxide production in LPS-stimulated RAW264.7 cells," Planta Medica, vol. 84, no. 18, pp. 1348-1354, 2018.

[23] C.-Y. Kuo, C.-H. Lin, and T. Hsu, "VHLInactivation in precancerous kidney cells induces an inflammatory response via ER stress-activated IRE1 $\alpha$ Signaling," Cancer Research, vol. 77, no. 13, pp. 3406-3416, 2017.

[24] M. J. Sung, M. Davaatseren, S. H. Kim, M. J. Kim, and J.-T. Hwang, "Boehmeria niveaattenuates LPS-induced inflammatory markers by inhibiting p38 and JNK phosphorylations in RAW264.7 macrophages," Pharmaceutical Biology, vol. 51, no. 9, pp. 1131-1136, 2013.

[25] O.-K. Kwon, M.-Y. Lee, J.-E. Yuk et al., “Anti-inflammatory effects of methanol extracts of the root of Lilium lancifolium on LPS-stimulated Raw264.7 cells," Journal of Ethnopharmacology, vol. 130, no. 1, pp. 28-34, 2010.

[26] K. Grynberg, F. Y. Ma, and D. J. Nikolic-Paterson, "The JNK signaling pathway in renal fibrosis," Frontiers in Physiology, vol. 8, p. 829, 2017.

[27] M. H. Park and J. T. Hong, "Roles of NF-kappaB in cancer and inflammatory diseases and their therapeutic approaches," Cells, vol. 5, no. 2, 2016.

[28] H.-f. Zhang, Y.-1. Wang, C. Gao et al., "Salvianolic acid a attenuates kidney injury and inflammation by inhibiting NF$\kappa \mathrm{B}$ and p38 MAPK signaling pathways in 5/6 nephrectomized rats," Acta Pharmacologica Sinica, vol. 39, no. 12, pp. 1855-1864, 2018.

[29] Z. Su, P. Yu, L. Sheng, J. Ye, and Z. Qin, "Fangjifuling ameliorates lipopolysaccharide-induced renal injury via inhibition of inflammatory and apoptotic response in mice," Cellular Physiology and Biochemistry, vol. 49, no. 6, pp. 2124-2137, 2018.

[30] F. Wu, D. S. Yao, T. Y. Lan et al., "Berberine prevents the apoptosis of mouse podocytes induced by TRAF5 overexpression by suppressing NF-kappaB activation," International Journal of Molecular Medicine, vol. 41, no. 1, pp. 555-563, 2017.

[31] K.-S. Oh, B. K. Oh, J. Mun, H. W. Seo, and B. H. Lee, "Salvianolic acid A suppress lipopolysaccharide-induced NF$\kappa \mathrm{B}$ signaling pathway by targeting IKK $\beta$," International Immunopharmacology, vol. 11, no. 11, pp. 1901-1906, 2011.

[32] J. Li, L. Bao, D. Zha et al., "Oridonin protects against the inflammatory response in diabetic nephropathy by inhibiting the TLR4/p38-MAPK and TLR4/NF- $\kappa$ B signaling pathways," International Immunopharmacology, vol. 55, pp. 9-19, 2018.

[33] W. Zheng, C.-Y. Zhou, X.-Q. Zhu et al., "Oridonin enhances the cytotoxicity of 5-FU in renal carcinoma cells by inducting necroptotic death," Biomedicine \& Pharmacotherapy, vol. 106, pp. 175-182, 2018.

[34] J. Zhou, E. J. Yun, W. Chen et al., "Targeting 3-phosphoinositide-dependent protein kinase 1 associated with drugresistant renal cell carcinoma using new oridonin analogs," Cell Death \& Disease, vol. 8, no. 3, Article ID e2701, 2017.

[35] E. Obeid, R. Nanda, Y.-X. Fu, and O. I. Olopade, "The role of tumor-associated macrophages in breast cancer progression," International Journal of Oncology, vol. 43, no. 1, pp. 5-12, 2013.

[36] N. Wang, H. Liang, and K. Zen, "Molecular mechanisms that influence the macrophage m1-m2 polarization balance," Frontiers in Immunology, vol. 5, p. 614, 2014.

[37] S. Kim, S. O. Ka, Y. Lee et al., "The new 4-O-methylhonokiol analog GS12021 inhibits inflammation and macrophage chemotaxis: role of AMP-activated protein kinase alpha activation," PLoS One, vol. 10, no. 2, Article ID e0117120, 2015.

[38] R. J. Davis, "Signal transduction by the JNK group of MAP kinases," Cell, vol. 103, no. 2, pp. 239-252, 2000.

[39] M. S. Han, T. Barrett, M. A. Brehm, and R. J. Davis, "Inflammation mediated by JNK in myeloid cells promotes the development of hepatitis and hepatocellular carcinoma," Cell Reports, vol. 15, no. 1, pp. 19-26, 2016.

[40] L. Chen, H. Deng, H. Cui et al., "Inflammatory responses and inflammation-associated diseases in organs," Oncotarget, vol. 9, no. 6, pp. 7204-7218, 2018. 